\title{
Oleaginous Yeasts: Biochemical Events Related with Lipid Synthesis and Potential Biotechnological Applications
}

\section{Seraphim Papanikolaou*}

Laboratory of Food Microbiology and Biotechnology, Department of Food Science and Technology, Agricultural University of Athens, 75 lera Odos, 11855 - Athens, Greece

The last years there has been a significant rise in the number of publications in the international literature that deal with the production of oils and fats deriving from microbial sources (the so called "single cell oils - SCOs") that could be used as precursors for the synthesis of bio-diesel or as "tailor-made" lipids amenable for the replacement of expensive fatty materials found in the plant or animal kingdom [1,2]. These lipids are produced by the so-called "oleaginous" microorganisms (microorganisms principally belonging to yeasts, fungi and algae and to lesser extent bacteria, capable of storing quantities of lipids higher than $20 \%$,wt/wt, in their dry weight) $[1,3-5]$.

Remarkable differences in biochemical and kinetic level exist between the process of lipid accumulation when glucose or similarly metabolized compounds are used as substrates ("de novo" lipid synthesis) compared with that performed when hydrophobic materials are used as substrates ("ex novo" lipid synthesis). De novo lipid biosynthesis in the oleaginous microorganisms is non-growth associated process, conducted due to change of intra-cellular concentration of various metabolites after nitrogen depletion into the culture medium. Nitrogen exhaustion leads to a rapid decrease of the concentration of cellular AMP, which is further cleaved in order for nitrogen to be offered to the microorganism. Cellular AMP concentration decrease alters the Krebs cycle function; $\mathrm{NAD}^{+}$- (and in various cases $\mathrm{NADP}^{+}$isocitrate) dehydrogenase, allosterically activated by intracellular AMP, loses its activity and the carbon flow, hence, is directed towards the accumulation of intra-mitochondrial citric acid. When the concentration of citric acid inside the mitochondria becomes higher than a critical value, it is secreted inside the cytoplasm. Then, citric acid is cleaved by ATP-citrate lyase, enzyme-key showing the oleaginous character of the microorganisms, into acetyl-CoA and oxaloacetate and acetyl-CoA, by virtue of the action of fatty acid synthetase generates cellular fatty acids and subsequently triacylglycerols (TAGs), that are the most common form of lipophilic compounds found in the oleaginous microorganisms [1,3-5]. In the non-lipid producing microorganisms, nitrogen exhaustions provokes secretion of the previously hyper-synthesized citric acid into the growth medium (case of the fungus Aspergillus niger and many of the strains of the yeast Yarrowia lipolytica) or results in a block in the level of 6-phosphofructokinase (with mechanisms similar to the ones related with the decrease of activity of $\mathrm{NAD}^{+}$-isocitrate dehydrogenase), leading in the accumulation of (intra-cellular) polysaccharides [1].

Lipid accumulation from hydrophobic substances (like TAGs, free fatty acids, soap-stocks, etc) used as the sole carbon and energy source (ex novo lipid accumulation) is performed by virtue of a radically different biochemical mechanism; culture TAGs are hydrolyzed with the aid of (extra-cellular or cell-bounded) lipases and fatty acids are incorporated inside the microbial cells or mycelia with various incorporation rates $[1,2]$. It is evident that while a relatively restricted number of microorganisms can grow on TAGs (these are the microorganisms that can synthesize lipases) much more microorganisms can present growth on media in which free fatty acids are used as the sole carbon source [1]. Substrate fatty acids are either assimilated for growth needs or become a substrate for intra-cellular bio-transformations $[1,2]$. "New" fatty acid profiles (in both extra- and intra-cellular level) that did not previously exist in the substrate fat are likely to be produced [1] (this is the so-called "fat biomodification process"). In the ex novo lipid accumulation process, lipid production is a growth associated process occurring simultaneously with cell growth, being entirely independent from nitrogen exhaustion from the culture medium $[1,2]$.

Oleaginous microorganisms routinely consume their own storage lipids when the flow rate of extra-cellular carbon source cannot cover the microbial metabolic requirements. Reserve lipid breakdown is independent from the carbon source that had previously been used for growth and lipid accumulation and occurs after rapid transition from carbon excess to carbon starvation conditions. In most cases, this process is accompanied by a significant lipid-free biomass production, showing that accumulated lipids act as endogenous carbon source for "new" cell material biosynthesis [1]. The process of accumulated lipid mobilization (turnover) is accompanied, in most of the cases, by a preferential degradation of the neutral accumulated lipid fractions (principally cellular TAGs). Furthermore, intra-cellular lipid turnover may result in a drastic modification of the fatty acid composition of the lipid, previously accumulated by the microorganism [1].

SCOs could constitute the starting material for the synthesis of the " $2^{\text {nd }}$ generation bio-diesel" (produced by virtue of the utilization of heterotrophic microorganisms that previously had accumulated lipid through fermentation of abundant sugar-based residues and renewable materials) or the " $3^{\text {rd }}$ generation bio-diesel" (produced via direct $\mathrm{CO}_{2}$ sequestration through the use of autotrophic oleaginous algae) [2]. It is obvious that as far as the $2^{\text {nd }}$ generation bio-diesel is concerned, only the conversion of sugars or related compounds in lipid is concerned; the ex novo lipid production process mainly refers to the utilization of lowcost hydrophobic materials as substrates, that through fermentation with the oleaginous microorganisms would be "up-graded", in order to finally present a "better" structure and/or composition compared with the initial fatty material $[1,2]$.

Yeasts, presenting a higher $\mu_{\max }$ and a more suitable morphology (single-cell development instead of pellets or mycelia) are microorganisms amenable for an easier handling in large-scale

*Corresponding author: Seraphim Papanikolaou, Laboratory of Food Microbiology and Biotechnology, Department of Food Science and Technology, Agricultural University of Athens, 75 lera Odos, 11855 - Athens, Greece, E-mail: spapanik@aua.gr

Received March 09, 2012; Accepted March 12, 2012; Published March 14, 2012

Citation: Papanikolaou S (2011) Oleaginous Yeasts: Biochemical Events Related with Lipid Synthesis and Potential Biotechnological Applications. Ferment Technol 1:e103. doi:10.4172/2167-7972.1000e103

Copyright: (C) 2011 Papanikolaou S. This is an open-access article distributed under the terms of the Creative Commons Attribution License, which permits unrestricted use, distribution, and reproduction in any medium, provided the original author and source are credited. 
cultivation conditions than the fungi, as far as many bio-processes including SCO fermentation are concerned $[1,3,4]$. A great number of scientific works refer to the utilization of yeasts belonging to the species Lipomyces starkeyi, Rhodosporidium toruloides, Apiotrichum curvatum (known also as Candida curvata or Cryptococcus curvatus), Rhodotorula spp., Trichosporon fermentans and Yarrowia lipolytica cultivated on several types of sugar-based or similarly metabolized raw materials, that include but are not limited to cheese-whey, glucose, glucose syrups, xylose, hemicellulose hydrolysates, molasses, waste glycerol deriving from bio-diesel production, organic acids, etc $[1,2,6]$, in order for bio-diesel precursors (e.g. TAGs) to be synthesized. However, it needs to be stressed that despite the large quantity of works recently appeared on this issue and although mass production of yeast lipids amenable for the synthesis of bio-diesel attracts currently noticeable interest, specifically at academic level, the production cost of SCOs remains always much higher than that of plant oils [6,7]. A representative value of yeast SCO produced on 2008 was around $3 \$$ per kg (excluding cost of feedstock used for SCO production) while the ones of rapeseed oil, soybean oil and sunflower oil were 1.4-1.5, 1.2-1.3 and 1.8-1.9 \$ per kg respectively [2,6]. However, the increment of bio-diesel needs, covered through the utilization of edible oils (this is the so-called " 1 st generation bio-diesel") has resulted in the fact that between 2007 and 2008 there has been a 2-fold increase of the price of conventional plant commodity oils [6]. Continuation of this situation and also severe rise in the price of crude oil (that currently is being reported) could potentially render the utilization of oleaginous yeasts as an economically viable process, in order for bio-diesel precursors to be produced [6,7].

A major aspect that could also be taken into consideration is that the economics of SCO bio-processes could be further ameliorated by using waste materials as substrates of the oleaginous microorganisms because most of these compounds have a negative value, their direct discharge without previous treatment causes serious environmental problems, while national or European legislation has rendered obligatory the treatment and safe disposal of these materials; for instance, the removal of non-toxic and non-hazardous waste material from the food industry (e.g. expired sugar-rich products, expired breads, concentrated sucrose- or glucose-based effluents, etc) that will further be subjected to compost processing costs $\sim 0.1-0.5$ US $\$$ per Kg of waste, depending on the nature of the food waste discarded $[1,8]$. One can imagine the economic losses for the food-processing facilities, specifically by taking into consideration that $\sim 20 \%$, wt/wt, of the materials utilized in these facilities finally turns to be converted into residues and the concomitant economic benefit by the establishment of sugar-based bio-refineries, so as to convert the residual materials into bio-diesel precursors [2]. On the other hand, successful utilization of waste streams has as prerequisite a well-established network providing the waste material into the fermentation plant and only under this circumstance the economic viability of the bio-refinery proposed could be secured [6]. Then the produced biomass rich in lipid can be directly trans-esterified to yield the bio-diesel [2], thus avoiding the oil extraction step, which is one of the most costly steps of the SCO production procedure [6], while the de-fatted yeast biomass could be used as feed supplement. However, in any case, a major problem that remains to be solved in order to significantly reduce the cost in the fermentation of SCO production is the one of the maintenance of aseptic conditions in the large-scale operations performed.

Another potential issue of the yeast lipids refers to their potentiality of "specialty-type" lipids production, which can possibly replace very expensive lipids found into the plant or animal kingdom. The price of various naturally occurring lipids and fats can tremendously vary (from 0.3 to up to 100 US \$ per kg - see: Ratledge and Wynn) [5]. Therefore identification of microorganisms capable of producing in increased quantities lipids with structure and composition similar to that of highvalue fats and subsequent large-scale production of these lipids can present an obvious financial interest, specifically if the starting material used is of low or negative cost. One such case refers to the replacement of the cocoa-butter by yeast lipids (the so-called microbial cocoabutter substitutes - CBSs). Cocoa-butter is commonly used in the food technology and principally in chocolate fabrication process, whereas it is also used in various cosmetology applications. It is principally produced in some of the African and Central American countries such as Ivory Coast, Nigeria, Jamaïca, etc. Its unique physicochemical characteristics (solid at ambient temperature but liquid at $37^{\circ} \mathrm{C}$ that of the temperature of mouth) are mainly due to the fatty acid composition of this fat; cocoa-butter is mainly composed of triacylglycerols of the type P-O-S and S-O-S (P: palmitic acid - C16:0, O: oleic acid $-{ }^{\Delta 9} \mathrm{C} 18: 1$, S: stearic acid - C18:0). Oleic acid, hence, is always found esterified in the position sn-2 of glycerol. Cocoa-butter contains $55-67 \% \mathrm{wt} / \mathrm{wt}$, saturated fatty acid while its composition is dependable on the plant variety and the culture conditions. An average fatty acid profile of this fat is: C16:0 23-30\% wt/wt; ${ }^{\Delta 9} \mathrm{C} 18: 130-37 \% \mathrm{wt} / \mathrm{wt}$; C18:0 32-37\% wt/wt; ${ }^{\Delta 9,12} \mathrm{C} 18: 22-4 \% \mathrm{wt} / \mathrm{wt}[2,4,5,8]$.

The production of CBSs, economically viable during the years 19801990 (at that time the price of the cocoa-butter was > 8.0 US \$ per kg), is obviously dependable of the price and the availability of this fat. During the years 1990-1994, a significant fall of cocoa-butter price $(<2.5$ US $\$$ per $\mathrm{kg}$ ) has constituted an enormous disadvantage for the production of various substitutes of this fat $[3,5,8]$. However, the years after 2000, there has been again an increase in the price of this lipid, due to the prevalence of harmful insects and viruses that have been reported to create several problems on cocoa-butter production $[2,5,8]$. Currently, the price of cocoa-butter is $\sim 5.0$ US $\$$ per Kg, though the tendency of this price, is to present (a remarkably significant) increase in the near future; an "extreme" scenario that recently has been presented in both the written and the electronic international press indicates that cocoabutter risks to disappear the next years due to general failure of the cultivation techniques of the cocoa plant and, thus, the utilization and application of the various CBSs will be generalized for the food industry [2].

By taking into consideration that most of the oleaginous yeast species during their conventional cultivation on glucose or similarly metabolized materials under nitrogen-limited conditions (as previously stated this is the major pre-requisite in order to "stress" the microorganism so as to accumulate fat) $[1,4,5]$ produce lipid that is essentially unsaturated while cocoa-butter is a fat principally saturated $[2,5]$, strategies have been developed in the literature in order to increase the content of cellular saturated fatty acids in the oleaginous yeasts; a first approach carried out in order to produce a microbial globally saturated lipid consisted of a conventional culture of oleaginous yeasts followed by a separation of the synthesized SCO. The result was revealed satisfactory considering both fusion point and organoleptic properties of the fabricated chocolate produced, though the inconvenient of this approach was that only the $35 \%$, wt/wt, of the produced SCO was found in the form S-U-S (S: Saturated fatty acid; U: Unsaturated fatty acid) [8]. A second approach utilized in order to increase the content of the yeast intra-cellular saturated fatty acids (principally that of C18:0) was the use of $\Delta 9$ and $\Delta 12$ desaturase inhibitors into the culture medium (e.g. sterculic acid and malvanic acid, that are cyclopropenic fatty acids found in the seed oil of different plants of the families Malvaceae 
Citation: Papanikolaou S (2011) Oleaginous Yeasts: Biochemical Events Related with Lipid Synthesis and Potential Biotechnological Applications. Ferment Technol 1:e103. doi:10.4172/2167-7972.1000e103

Page 3 of 3

and Sterculiaceae). Being fatty acids of 17 or 18 carbon atoms with a cyclopropenic group in $\Delta 9$ position, the above-mentioned compounds constitute desaturase inhibitors and, indeed, although in some cases significant quantities of the cyclopropenic inhibitor had been added into the culture medium (e.g. until $2 \mathrm{~mL} / \mathrm{L}$ of sterculia oil), cell growth and lipid accumulation have not been altered at all, while significant modification of the reserve lipids was observed; decreased amounts of oleic acid were detected whilst a significant rise of the stearic acid amount was observed $[4,5,8]$. Although yeast lipids with composition similarities with the cocoa-butter have been synthesized, this strategy presents two fundamental drawbacks that are the increased cost of the inhibitors used and the fact that cyclopropenic inhibitors are potential carcinogenic compounds [2].

A genetic manipulation strategy based on the deletion of the gene encoding for $\Delta 9$ dehydrogenase, which is the responsible enzyme for the biotransformation of stearoyl-CoA to oleoyl-CoA, was also applied in order for the production of CBSs to be performed [3-5]. Cellular suspensions of the oleaginous wild type A. curvatum have been treated with mutagenic factors and compared with the lipid profile of the wild type, auxotrophic unsaturated fatty acid ( $U f a)$ mutants have presented a significant rise in the quantity of cellular C18:0 amount $[2,3,8]$. Moreover, another strategy that has been developed was based exactly on the same assumption with the previous "genetic engineering" approach. The assumption was based on the fact that all of the reactions of desaturation in the nature are oxygen-dependent. Therefore, a critical limitation of the oxygenation in the fermentation medium could decrease the conversion percentage of stearoyl-CoA to oleoylCoA without substantially inhibiting the microbial growth and the process of lipid accumulation. Indeed, although the amount of stearic acid into the reserve lipids was not very high, the sum of saturated fatty acids (e.g. 16:0, 18:0, 20:0 and 24:0) was most of the cases higher than $50 \%, \mathrm{wt} / \mathrm{wt}$, of total lipids, a quantity similar to that of the cocoa-butter, while this approach was the only one extrapolated in semi-industrial (500-L bubble column reactors) and finally to industrial $\left(13-\mathrm{m}^{3}\right.$ reactors) scale $[2,4,5]$.

Finally, one of the common approaches practiced, was the growth of oleaginous yeasts in preparations of pure stearic acid or its derivatives, in order to direct the cellular metabolism towards the accumulation of lipid rich in C18:0 [2,8]. While the utilization of pure stearic acid is expensive, utilization of hydrophobic by-products or waste streams composed of saturated fatty acids (e.g. stearin that is an industrial derivative of tallow composed principally of C18:0) is not [8]. Correct use of this inexpensive fatty by-product of the meat-processing industry together with another cheap donor of the fatty acid ${ }^{\Delta 9} \mathrm{C} 18: 1$ (e.g. hydrolyzed rapeseed oil or waste glycerol deriving from bio-diesel units) by the yeast $Y$. lipolytica, resulted in the synthesis of CBSs $[2,8]$.

The production of lipid from microbial sources presents continuous expansion in the last years. Amongst microbial lipids, yeast lipids present a very high importance in both academic and industrial point of view. Due to their unicellular nature and their potentiality to grow on a plethora of hydrophilic or hydrophobic substrates, the oleaginous yeasts are considered as perfect "tools" for studying phenomena of advanced lipid biochemistry and biotechnology $[1,3,5,8]$. The last years, works indicating interesting analogies between lipid metabolism in the yeast cells and the gastro-intestinal tract and vascular system have appeared [9]. Also, increasing demand and utilization of $1^{\text {st }}$ generation bio-diesel has increased the cost of various food-stuffs and this led to the necessity of discovery of non-conventional sources of oils, that could be subsequently converted into bio-diesel. The oleaginous yeasts, which in most cases are categorized as GRAS microorganisms and due to their fast growth rates and their potentiality to grow on a plethora of substrates, are considered perfect candidates for the production of this $2^{\text {nd }}$ generation bio-diesel. Finally, the potential (significant) rise in the price of cocoa-butter or the already existing high price of other saturated exotic fats (e.g. shea butter, sal fat) renders the utilization of several oleaginous yeast strains amenable to produce substitute SCOs of these high-value fats as very promising.

\section{Referrences}

1. Papanikolaou S, Aggelis G (2011) Lipids of oleaginous yeasts. Part I: Biochemistry of single cell oil production. Eur J Lipid Sci Technol 113: 10311051.

2. Papanikolaou S, Aggelis G (2011) Lipids of oleaginous yeasts. Part II Technology and potential applications. Eur J Lipid Sci Technol 113: 1052-1073.

3. Ratledge C (1997) Microbial lipids. In: Biotechnology, $2^{\text {nd }}$ edition, volume 7 . Kleinkauf H, Dohren H (Eds). VCH Weinheim - Germany, 135-197.

4. Davies RJ, Holdsworth JE (1992) Synthesis of lipids in yeasts: biochemistry, physiology and production. Adv Appl Lipid Res 1: 119-159.

5. Ratledge C, Wynn JP (2002) The biochemistry and molecular biology of lipid accumulation in oleaginous microorganisms. Adv Appl Microbiol 51: 1-51.

6. Ratledge C, Cohen Z (2008) Microbial and algal lipids: do they have a future for biodiesel or as commodity oils? Lipid Technol 20: 155-160.

7. Ratledge C (2011) Are algal oils realistic options for biofuels? Eur J Lipid Sci Technol 113: 135-136.

8. Papanikolaou S, Aggelis G (2010) Yarrowia lipolytica: a model microorganism used for the production of tailor-made lipids. Eur J Lipid Sci Technol 112: 639654

9. Pembroke $T$ (2006) The yeast Yarrowia lipolytica as a model for obesity. Nat Inst Health Sci Res Bull 3: 11-13. 